\title{
Microscopic Linear Response Theory of Spin Relaxation and Relativistic Transport Phenomena in Graphene
}

\author{
Manuel Offidani ${ }^{1}(\mathbb{D})$, Roberto Raimondi ${ }^{2, *(D)}$ and Aires Ferreira ${ }^{1, *(D)}$ \\ 1 Department of Physics, University of York, York YO10 5DD, UK; manuel.offidani@york.ac.uk \\ 2 Dipartimento di Matematica e Fisica, Università Roma Tre, 00146 Rome, Italy \\ * Correspondence: roberto.raimondi@uniroma3.it (R.R.); aires.ferreira@york.ac.uk (A.F.)
}

Received: 23 April 2018; Accepted: 18 May 2018; Published: 22 May 2018

\begin{abstract}
We present a unified theoretical framework for the study of spin dynamics and relativistic transport phenomena in disordered two-dimensional Dirac systems with pseudospin-spin coupling. The formalism is applied to the paradigmatic case of graphene with uniform Bychkov-Rashba interaction and shown to capture spin relaxation processes and associated charge-to-spin interconversion phenomena in response to generic external perturbations, including spin density fluctuations and electric fields. A controlled diagrammatic evaluation of the generalized spin susceptibility in the diffusive regime of weak spin-orbit interaction allows us to show that the spin and momentum lifetimes satisfy the standard Dyakonov-Perel relation for both weak (Gaussian) and resonant (unitary) nonmagnetic disorder. Finally, we demonstrate that the spin relaxation rate can be derived in the zero-frequency limit by exploiting the $\mathrm{SU}(2)$ covariant conservation laws for the spin observables. Our results set the stage for a fully quantum-mechanical description of spin relaxation in both pristine graphene samples with weak spin-orbit fields and in graphene heterostructures with enhanced spin-orbital effects currently attracting much attention.
\end{abstract}

Keywords: graphene; spintronics; spin relaxation; 2DEGs; diagrammatic theory; spin-Galvanic effect; spin-orbit coupling

\section{Introduction}

\subsection{Spin Relaxation in Graphene}

Graphene is considered a promising material for spintronics applications due to its negligible hyperfine interactions and low spin-orbit coupling (SOC) [1,2]. Early theoretical estimates hinted at ultra-long spin lifetime ( $\tau_{s} \approx 1-100 \mu \mathrm{s}$ ) [3], whereas experiments found $\tau_{s}$ to be limited to a few nanoseconds [4]. The microscopic mechanisms responsible for the relatively fast spin relaxation in high-mobility graphene samples remain controversial [5], but recent findings indicate that spinful scatterers, such as magnetic adatoms, are the primary cause of spin relaxation [6-9].

The spin dynamics in graphene is conventionally probed by means of nonlocal transport measurements $[10,11]$. In this approach, a spin current is injected from ferromagnetic electrodes into the graphene channel and allowed to diffuse under the effect of a perpendicular magnetic field. The Larmor precession of the electrons' spin about the external field modulates the average spin accumulation detected away from the injection point (Hanle curve), resulting in a bona fide spin signal from which $\tau_{s}$ can be deduced. Such Hanle precession measurements found a large spread in $\tau_{s}$ from tens of picoseconds up to a few nanoseconds [12-22], reflecting the different sample preparation and device fabrication methods. Theoretical studies have revealed a number of possible 
spin relaxation sources, including magnetic impurities, spin-orbit active adatoms, ripples and other substrate effects [23-30]. Numerical approaches have provided further insight into the relaxation mechanisms, enriching the scenario to include the impact of electron-hole puddles, pseudospin-spin coherence and ballistic effects [31-33]. Despite the relatively short $\tau_{s}$ of clean samples, the high charge carrier mobility allows spins to diffuse over extremely long distances up to $13 \mu \mathrm{m}$ at room temperature [34-36].

The paradigmatic model for studies of spin relaxation in graphene is the two-dimensional (2D) Hamiltonian of massless Dirac fermions supplemented with a (uniform or random) Bychkov-Rashba interaction [37]. This type of SOC has its origin in perturbations breaking the inversion symmetry, which include substrate-induced electric fields, adatoms, and ripple-induced gauge fields [3,4]. The Bychkov-Rashba interaction in graphene (hereafter referred to as Rashba SOC) can be seen as a non-Abelian gauge field that couples to the intrinsic pseudospin of Dirac fermions, enabling spin relaxation upon impurity scattering, e.g., via the familiar Dyakonov-Perel (DP) mechanism [38].

Graphene with random Rashba SOC has been recently shown to host novel charge-to-spin conversion effects by means of a quantum extension of the Boltzmann transport theory [39,40]. Previous theoretical descriptions of spin relaxation in such 2D Dirac models with uniform Rashba interaction were instead based on semiclassical approximations [41,42]. On the other hand, a fully quantum-mechanical theory of spin-orbit-coupled transport for 2D Dirac-Rashba systems in the static (DC) limit has been formulated recently by the authors [43,44]. Analogously to the 2D electron gas (2DEG) case [45-47], it was shown that impurity scattering corrections exactly balance the intrinsic generation of a spin Hall current for spin-independent disorder, $\left\langle\mathcal{J}_{\mathrm{SH}}\right\rangle_{\mathcal{E}}=0$, where $\mathcal{E}$ is an external DC electric field [43]. The vanishing of the spin Hall effect in this model is connected to the establishment of a robust nonequilibrium in-plane spin polarization $\langle\mathbf{S}\rangle_{\mathcal{E}} \neq 0$ with $\mathbf{S} \perp \mathcal{E}$, known as inverse spin-Galvanic effect (ISGE) [44]. However, a time-dependent framework able to unveil how the steady state is reached within the 2D Dirac-Rashba model is yet to be developed. In this paper, we address this problem. We derive the coupled spin-charge drift-diffusion equations for nonmagnetic disorder and generic homogeneous perturbations by means of the diagrammatic technique for disordered electrons. A similar approach has been adopted very recently in the context of 2DEGs with both Bychkov-Rashba and Dresselhaus interactions [48], where it was shown perfect agreement between the Kubo diagrammatic formalism and the Keldysh SU(2) gauge theory [49]. In this work, we extend the standard quantum diagrammatic formalism to accommodate the enlarged 2 (spin) $\otimes 2$ (pseudospin) Clifford structure of the 2D Dirac-Rashba model leading to a 16-dimensional diffuson operator in the absence of intervalley scattering. We find that the typical DP relation connecting the spin relaxation time (SRT) and the momentum lifetime in the weak SOC regime, that is $\tau_{S} \propto \tau^{-1}$ for $\lambda \tau \ll 1$, where $\lambda$ is the SOC strength, holds at all orders in the scattering potential strength. The meaning and interpretation of our results for the SRTs can be also clarified by the SU(2) covariant conservation laws inherent to the diagrammatic (perturbative) structure, whose usage allows us derive the DP relation even in the zero-frequency limit. In particular, we provide the analytical expression of $\tau_{s}$ in the unitary limit of very strong potential scattering.

\subsection{Dirac-Rashba Model}

The effective low-energy Hamiltonian describing the electronic properties of 2D Dirac fermions subject to a uniform Rashba interaction around the $K$ point reads as [50]

$$
H=\int d \mathbf{x} \Psi^{\dagger}(\mathbf{x})[v \sigma \cdot \mathbf{p}+\lambda(\sigma \times \mathbf{s}) \cdot \hat{z}+V(\mathbf{x})] \Psi(\mathbf{x}),
$$

where $v$ is the bare velocity of massless Dirac fermions, $\mathbf{p}=-\imath \boldsymbol{\nabla}$ is the 2D kinematic momentum operator, $\lambda$ is the SOC strength and $\sigma_{i}, s_{i}(i=x, y, z)$ are Pauli matrices associated with sublattice (pseudospin) and spin degrees of freedom, respectively. Here, $V(\mathbf{x})$ is a disorder potential describing elastic scattering from nonmagnetic short-range impurities. For simplicity, in this work, we neglect 
intervalley scattering processes, which in the pure Rashba model can renormalize the momentum lifetime but are not expected to impact fundamentally the spin dynamics [43]. Thus, it suffices to consider the low-energy dynamics around the $K$ point.

The energy dispersion relation of the free Hamiltonian $H_{0}=H-V$ in Equation (1) is

$$
\epsilon_{\mu \nu}(\mathbf{k})=\mu \lambda+v \sqrt{\lambda^{2}+v^{2}|\mathbf{k}|^{2}}
$$

where $\mu, v= \pm 1$ labels the various subbands (Figure 1a).

(a)

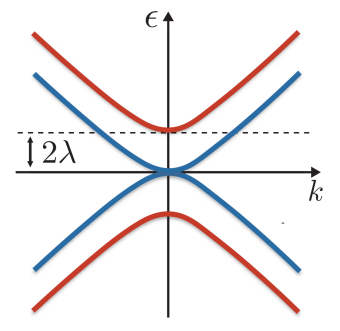

(b)

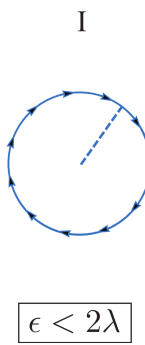

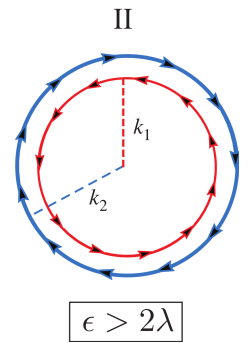

Figure 1. (a) Energy dispersion around the $K$ point. The splitting of the Dirac bands leads to a spin gap or pseudogap. (b) Tangential winding of the spin texture in regimes I and II.

The Rashba interaction aligns the electron spin at right angles to the wavevector, the so-called spin-momentum locking configuration (Figure 1b) [51,52]. For Fermi energy $|\epsilon|>2|\lambda|$ (region II), the split Fermi surface displays counter-rotating spin textures reminiscent of (nonchiral) 2DEGs with Rashba interaction [37]. A regime (pseudogap, region I) where the Fermi energy intersects a single subband, with electronic states having well-defined spin helicity, extends for energies $|\epsilon|<2|\lambda|$. In the conventional 2DEG this circumstance only happens at a single point, i.e., the intersection between the parabolic bands [53]. Importantly, the spin texture of energy bands in the 2D Dirac-Rashba model is modulated by the band velocity, i.e.,

$$
\langle\mathbf{s}\rangle_{\mu \nu \mathbf{k}}=-\mu\langle\sigma\rangle_{\mu \nu \mathbf{k}} \times \hat{\mathbf{z}}
$$

where $\langle\sigma\rangle_{\mu v \mathbf{k}}=(\nu / v) \nabla_{\mathbf{k}} \epsilon_{\mu v}(\mathbf{k})$ is the pseudospin polarization vector. The entanglement between pseudospin and spin degrees of freedom in the model is responsible for a rich energy dependence of transport coefficients $[43,44]$. For brevity of notation, we assume $\epsilon, \lambda>0$ in the remainder of the work.

\subsection{Disorder Effects}

The random potential in Equation (1) affects the spin dynamics by inducing elastic transitions between electronic states $(\mu \nu \mathbf{k}) \rightarrow\left(\mu^{\prime} v^{\prime} \mathbf{k}^{\prime}\right)$ associated with different effective Larmor fields, $\boldsymbol{\Omega}_{\mu v \mathbf{k}}=$ $\lambda\langle\mathbf{s}\rangle_{\mu \nu \mathbf{k}} \approx-\mu \nu \lambda \hat{\mathbf{k}} \times \hat{z}$ for $\epsilon \gg \lambda$. This random change in the spin precession axis is responsible for the irreversible loss of spin information. To describe the effects of disorder, we employ standard many-body perturbation theory methods. We work within the zero-temperature Green's function formalism.

The retarded ( $\mathrm{R})$ /advanced (A) single-particle Green's function $(a=A, R \equiv-,+)$ is

$$
G^{a}\left(\mathbf{x}, \mathbf{x}^{\prime} ; t-t^{\prime}\right)=\mp \imath\left\langle 0\left|\mathcal{T}\left[\Psi(\mathbf{x}, t), \Psi^{\dagger}\left(\mathbf{x}^{\prime}, t^{\prime}\right)\right]\right| 0\right\rangle \theta\left( \pm t \mp t^{\prime}\right),
$$

where $\mathcal{T}$ is the time-ordering symbol and $\theta($.$) is the Heaviside step function. Changing to the energy$ domain, one obtains

$$
G^{a}\left(\mathbf{x}, \mathbf{x}^{\prime} ; \epsilon\right)=\left\langle\mathbf{x}^{\prime}\left|\frac{1}{\left[G_{0}^{a}(\epsilon)\right]^{-1}-V}\right| \mathbf{x}\right\rangle,
$$


where $G_{0}^{a}(\epsilon)=\left(\epsilon+\imath v \sigma \cdot \nabla+\lambda(\sigma \times \mathbf{s}) \cdot \hat{z} \pm \imath 0^{+}\right)^{-1}$ is the Green's function of free 2D Dirac-Rashba fermions (see Appendix A).

The central quantity in our approach is the disorder averaged Green's function, $\mathcal{G}^{a}\left(\mathbf{x}-\mathbf{x}^{\prime}, \epsilon\right)=$ $\overline{G^{a}\left(\mathbf{x}^{\prime} \mathbf{x}^{\prime} ; \epsilon\right)}$, where the bar - denotes the average over all impurity configurations (Figure 2a). Its momentum representation is

$$
\mathcal{G}_{\mathbf{k}}^{a}(\epsilon)=\frac{1}{\left[\mathcal{G}_{0 \mathbf{k}}^{a}(\epsilon)\right]^{-1}-\Sigma_{\mathbf{k}}^{a}(\epsilon)},
$$

where $\mathcal{G}_{0 \mathbf{k}}^{a}(\epsilon)$ is the Fourier transform of $G_{0}^{a}\left(\mathbf{x}-\mathbf{x}^{\prime} ; \epsilon\right)$ and

$$
\Sigma_{\mathbf{k}}^{a}(\epsilon)=\int d\left(\mathbf{x}-\mathbf{x}^{\prime}\right) e^{-l \mathbf{k}\left(\mathbf{x}-\mathbf{x}^{\prime}\right)} \overline{\left\langle\mathbf{x}^{\prime}\left|V \frac{1}{1-G_{0}^{a}(\epsilon) V}\right| \mathbf{x}\right\rangle}
$$

is the disordered averaged self-energy within the noncrossing approximation. The latter neglects coherent multiple impurity scattering corrections, which is justified in the diffusive regime with $\epsilon \tau \gg 1$ [54]. The self-energy induced by short-range impurities is k-independent, $\Sigma_{\mathbf{k}}^{a}(\epsilon) \equiv \Sigma^{a}(\epsilon)$, and hence we drop this index in what follows.

(a)

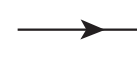

(b)

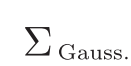

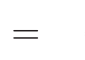
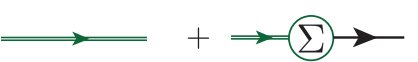

(c) $\quad \Sigma_{\text {TMA }}$

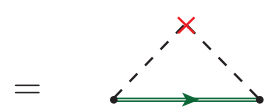

$=\begin{gathered}x \\ 1 \\ \vdots \\ 1\end{gathered}+$
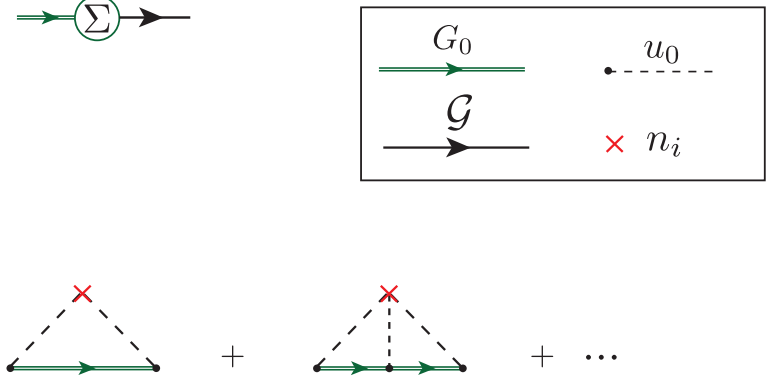

Figure 2. (a) Dyson equation for the disordered averaged Green's function; and $(\mathbf{b}, \mathbf{c})$ approximation schemes for evaluation of the self-energy: Gaussian (b); and T-matrix approximation (TMA) (c). Box shows Feynman rules for the disorder potential insertions (dashed lines) and impurity density insertion (red crosses).

To account for the characteristic resonant (unitary) scattering regime of graphene with relaxation time $\tau \propto \epsilon[55,56]$, we adopt a $T$-matrix approach by evaluating the self-energy $\Sigma^{a}(\epsilon)$ at all orders in $V$. We obtain

$$
\Sigma^{a}(\epsilon)=n_{i} \frac{u_{0}}{1-u_{0} g_{0}^{a}(\epsilon)}+O\left(n_{i}^{2}\right)=n_{i} T^{a}(\epsilon),
$$

where $u_{0}$ parameterizes the scattering strength of the spin-transparent (scalar) impurities, $n_{i}$ is the impurity areal density and $T^{a}(\epsilon)$ is the single-impurity $T$-matrix. Note that multiple impurity scattering diagrams $\propto O\left(n_{i}^{2}\right)$ can be neglected in the limit $\epsilon \tau \gg 1$, i.e., away from the Dirac point (refer to Section 2.4 for a brief discussion of the spin relaxation within the full noncrossing approximation). We have also introduced

$$
g_{0}^{a}(\epsilon)=g_{0,0}^{a}(\epsilon) \gamma_{0}+g_{0, z z}^{a}(\epsilon) \gamma_{z z}+g_{0, r}^{a}(\epsilon) \gamma_{r},
$$


as the momentum integrated Green's function of the clean system (cf. Equation (A1) of Appendix A), where $\gamma_{0} \equiv \sigma_{0} s_{0}$ is the $4 \times 4$ identity matrix, $\gamma_{z z}=\sigma_{z} \mathcal{s}_{z}, \gamma_{r}=(\sigma \times \mathbf{s})_{z}$ and

$$
\begin{aligned}
g_{0,0}^{a}(\epsilon) & =-\frac{1}{8 \pi v^{2}}\left[\epsilon\left(\mathcal{L}_{\mathrm{II}}(\epsilon)+a \imath \pi \theta_{\mathrm{II}}(\epsilon)\right)+\lambda\left(\mathcal{L}_{\mathrm{I}}(\epsilon)+a \imath \pi \theta_{\mathrm{I}}(\epsilon)\right)\right], \\
g_{0, z z}^{a}(\epsilon) & =-\frac{\lambda}{8 \pi v^{2}}\left(\mathcal{L}_{\mathrm{I}}(\epsilon)+a \imath \pi \theta_{\mathrm{I}}(\epsilon)\right), \\
g_{0, r}^{a}(\epsilon) & =+\frac{\epsilon}{16 \pi v^{2}}\left(\mathcal{L}_{\mathrm{I}}(\epsilon)+a \imath \pi \theta_{\mathrm{I}}(\epsilon)\right) .
\end{aligned}
$$

In the above, $\theta_{\mathrm{I}(\mathrm{II})}(\epsilon)=\theta(\epsilon+2 \lambda) \mp \theta(\epsilon-2 \lambda)$ selects the energy regime and

$$
\mathcal{L}_{\mathrm{I}(\mathrm{II})}(\epsilon)=\log \left|\frac{\Lambda^{2}}{\epsilon(\epsilon+2 \lambda)}\right| \mp \log \left|\frac{\Lambda^{2}}{\epsilon(\epsilon-2 \lambda)}\right|,
$$

with $\Lambda$ denoting the ultraviolet cutoff of the low-energy theory [55].

The self-energy simplifies in two important limiting cases: (i) weak Gaussian disorder $\left(\left|u_{0}\right| \ll\left|g_{0}^{a}\right|^{-1}\right)$; and (ii) unitary disorder $\left(u_{0} \rightarrow \pm \infty\right)$. In the weak scattering regime, it suffices to only take into account the "rainbow" diagram with two impurity lines in the Dyson expansion (see Figure 2b). For scalar disorder, this approximation is equivalent to assuming that the disorder potential satisfies white-noise statistics [54]

$$
\begin{aligned}
\langle V(\mathbf{x})\rangle & =0, \\
\left\langle V(\mathbf{x}) V\left(\mathbf{x}^{\prime}\right)\right\rangle & =n_{i} u_{0}^{2} \delta\left(\mathbf{x}-\mathbf{x}^{\prime}\right) .
\end{aligned}
$$

In this case, we have

$$
\left.\Sigma^{a}(\epsilon)\right|_{\text {Gauss. }}=n_{i} u_{0}^{2} g_{0}^{a}(\epsilon)
$$

The real part of the self-energy provides a parametrically small renormalization of the band structure, which can be safely neglected in the diffusive regime of interest [43]. We thus find

$$
\Sigma^{R / A}=\mp i n_{i}\left(\eta_{0} \gamma_{0}+\eta_{r} \gamma_{r}+\eta_{z z} \gamma_{z z}\right),
$$

where the functions $\eta_{0}, \eta_{r}, \eta_{z z}$, proportional to the imaginary parts of Equations (10)-(12), have different forms depending on the Fermi level position. In this work, we restrict the analysis to diffusive systems with weak SOC $\lambda \tau \ll 1$ and $\epsilon \gg \lambda$. It is thus convenient to express the various quantities in $\Sigma^{a}(\epsilon)$ in terms of the quasiparticle broadening in regime II, i.e.,

$$
\left.\frac{1}{2 \tau} \equiv n_{i} \eta_{0}\right|_{\epsilon>2 \lambda}
$$

Explicitly, we have

$$
\left.\frac{1}{2 \tau}\right|_{\text {Gauss. }}=n_{i} \frac{u_{0}^{2} \epsilon}{4 v^{2}}, \eta_{z z}=0, \eta_{r}=0
$$

For a typical choice of parameters, say, $n_{i}=10^{12} \mathrm{~cm}^{-2}, u_{0}=1\left(u_{0}\right.$ is in units of eV.nm $\left.{ }^{-2}\right)$ and $\epsilon=50 \mathrm{meV}$, one finds $\left.\tau\right|_{\text {Gauss }} \simeq 1.14 \mathrm{ps}$, which is representative of clean graphene samples [55].

Within the $T$-matrix formalism, the nontrivial part of $\Im \Sigma^{a}(\epsilon)$ acquires a finite value. However, in the unitary limit of strong potential scattering $\left(u_{0} \rightarrow \infty\right)$, we have $\Sigma^{a}(\epsilon)=-n_{i} / g_{0,0}^{a}(\epsilon)$ and we recover a scalar self-energy, with

$$
\left.\frac{1}{2 \tau}\right|_{\mathrm{TMA} ; u \rightarrow \infty}=\frac{n_{i}}{\epsilon} \frac{4 \pi^{2} v^{2}}{\pi^{2}+\mathcal{L}_{\mathrm{II}}^{2}(\epsilon)}, \eta_{z z}=0, \eta_{r}=0
$$


In this case, considering $\lambda=10 \mathrm{meV}, \Lambda=10 \mathrm{eV}$ and $n_{i}, \epsilon$ as above, one obtains a substantially shorter scattering time $\left.\tau\right|_{\text {TMA }}=0.08$ ps. The unitary result captures the typical energy dependence $\tau \propto \epsilon$ observed in high-mobility graphene samples [55], where the charge carrier mobility is likely limited by short-range scatterers, including adsorbates, short-range ripples and vacancies [57-60].

\section{Microscopic Linear Response Theory for Spin Relaxation}

\subsection{General Formalism}

We consider the long-wavelength spin dynamics generated by a generic external perturbation

$$
H_{\alpha \beta}^{\text {ext }}(\mathbf{x}, t)=-\mathcal{J}_{\alpha \beta} \mathcal{A}_{\alpha \beta}(\mathbf{x}, t),
$$

where $\mathcal{J}_{\alpha \beta} \propto \sigma_{\alpha} s_{\beta}(\alpha, \beta=0, i)$ is the current density operator $(\alpha=x, y)$ or density operator $(\alpha=0, z)$ and $\mathcal{A}_{\alpha \beta}$ is a generalized vector potential [43]. We consider in detail two important cases: (i) an electric field perturbation e.g., $H_{x 0}^{\text {ext }}(\mathbf{x}, t)=-v \sigma_{x} s_{0} A_{x}(\mathbf{x}, t)$; and (ii) a spin density fluctuation $H_{0 i}^{\text {ext }}(\mathbf{x}, t)=$ $-\frac{1}{2} \sigma_{0} s_{i} B_{i}(\mathbf{x}, t)$. The induced spin polarization density

$$
S_{i}(\mathbf{x}, t)=\frac{1}{2}\left\langle\Psi^{\dagger}(\mathbf{x}, t) \sigma_{0} s_{i} \Psi(\mathbf{x}, t)\right\rangle
$$

is evaluated within the framework of linear response theory. This approach has been applied to derive charge-spin diffusion equations describing spin dynamics and magnetoelectric effects in 2DEGs $[48,61,62]$. As shown below, a suitable extension of this approach to accommodate the enlarged (spin $\otimes$ pseudospin) Clifford algebra $\gamma_{\alpha \beta}=\sigma_{\alpha} s_{\beta}$ will allow us to obtain a rigorous microscopic theory of diffusive transport and spin relaxation for 2D Dirac systems.

The linear response of the $\hat{i}$-component of the spin polarization vector at zero temperature reads as

$$
S_{i}(\mathbf{x}, t)=-\int d \mathbf{x}^{\prime} \int_{-\infty}^{\infty} d t^{\prime} \chi_{i, \alpha \beta}\left(\mathbf{x}-\mathbf{x}^{\prime}, t-t^{\prime}\right) \partial_{t^{\prime}} \mathcal{A}_{\alpha \beta}\left(\mathbf{x}^{\prime}, t^{\prime}\right),
$$

where $\chi_{i, \alpha \beta}\left(\mathbf{x}-\mathbf{x}^{\prime}, t-t^{\prime}\right)$ is the generalized spin susceptibility associated to the external perturbation, i.e., an electric field $\mathcal{E}_{x}(\mathbf{x}, t)=-\partial_{t} A_{x}(\mathbf{x}, t)$ or a "spin injection field" $\Phi_{i}(\mathbf{x}, t)=-\partial_{t} B_{i}(\mathbf{x}, t)$ [63]. Expressing the above equation in terms of the Fourier transform $\chi_{i, \alpha \beta}(\mathbf{q}, \omega)$ in the long-wavelength limit $\mathbf{q} \rightarrow 0$, we have

$$
\chi_{i, \alpha \beta}(0, \omega)=\frac{\kappa}{2} \operatorname{Tr}\left\langle\overline{\gamma_{0 i} G^{R}\left(\mathbf{x}, \mathbf{x}^{\prime} ; \epsilon+\omega\right) \gamma_{\alpha \beta} G^{A}\left(\mathbf{x}^{\prime}, \mathbf{x} ; \epsilon\right)}\right\rangle,
$$

where $\kappa=v(\kappa=1 / 2)$ for a electric (spin injection) field and $\operatorname{Tr}$ is the trace over all degrees of freedom. Terms involving products of Green's functions on the same sector (RR and AA) are smaller by a factor of $(\epsilon \tau)^{-1}$ and thus can be safely neglected.

The disorder average in Equation (24) is evaluated by means of the diagrammatic technique (Figure 3). For brevity of notation, we first present the formalism within the Gaussian approximation for the self-energy, Equation (16). In Section 2.3, we provide the connection with the full T-matrix result.

A summation of noncrossing two-particle (ladder) diagrams leads to

$$
\chi_{i, \alpha \beta}^{(\mathrm{NC})}(0, \omega)=\frac{\kappa}{2} \sum_{\mathbf{k}} \operatorname{tr}\left\{\gamma_{0 i} \mathcal{G}_{\mathbf{k}}^{R}(\epsilon+\omega) \tilde{\gamma}_{\alpha \beta}(\omega) \mathcal{G}_{\mathbf{k}}^{A}(\epsilon)\right\}
$$

where $\operatorname{tr}$ is the trace over internal degrees of freedom (spin and sublattice). The dressed vertex $\tilde{\gamma}_{\alpha \beta}$ satisfies the Bethe-Salpeter (BS) equation

$$
\tilde{\gamma}_{\alpha \beta}(\omega)=\gamma_{\alpha \beta}+\frac{4}{2 \pi \tau N_{0}} \sum_{\mathbf{k}} \mathcal{G}_{\mathbf{k}}^{R}(\epsilon+\omega) \tilde{\gamma}_{\alpha \beta}(\omega) \mathcal{G}_{\mathbf{k}}^{A}(\epsilon) .
$$


where $N_{0} \equiv \epsilon / \pi v^{2}$ (for the $T$-matrix extension see Equation (59) and text therein). Projecting onto the elements of the Clifford algebra

$$
\tilde{\gamma}_{\alpha \beta \varrho \zeta}(\omega)=\frac{1}{4} \operatorname{tr}\left[\tilde{\gamma}_{\alpha \beta}(\omega) \sigma_{\varrho} S_{\zeta}\right],
$$

we recast the BS equation into the form

$$
\tilde{\gamma}_{\alpha \beta \varrho \varsigma}(\omega)=\delta_{\alpha \varrho} \delta_{\beta \varsigma}+\sum_{\mu, v=0, x, y, z} M_{\mu v \varrho \varsigma}(\omega) \tilde{\gamma}_{\alpha \beta \mu \nu}(\omega),
$$

where

$$
M_{\mu \nu \varrho \varsigma}(\omega)=\frac{1}{2 \pi \tau N_{0}} \sum_{\mathbf{k}} \operatorname{tr}\left[\mathcal{G}_{\mathbf{k}}^{R}(\epsilon+\omega) \gamma_{\mu \nu} \mathcal{G}_{\mathbf{k}}^{A}(\epsilon) \gamma_{\varrho \varsigma}\right]
$$

Introducing the 16-dimensional vectors $\tilde{\gamma}_{\alpha \beta}(\omega)=\left(\tilde{\gamma}_{\alpha \beta 00}(\omega), \ldots, \tilde{\gamma}_{\alpha \beta z z}(\omega)\right)^{\mathrm{t}}$ and $\gamma_{\alpha \beta}=$ $\left(0,0, \ldots, \gamma_{\alpha \beta \alpha \beta}, \ldots, 0\right)^{\mathrm{t}}$ a more compact matrix form for Equation (26) is given in terms of the diffusion operator $\mathcal{D}^{-1}$ as

$$
\mathcal{D}^{-1} \tilde{\gamma}_{\alpha \beta}(\omega) \equiv\left(\mathbf{1}_{16 \times 16}-M^{\mathrm{t}}(\omega)\right) \tilde{\gamma}_{\alpha \beta}(\omega)=\gamma_{\alpha \beta} .
$$

The spin relaxation rates are simply identified as the poles of the generalized susceptibility in the complex $\omega$-plane. The determination of the SRTs is thus reduced to the analysis of the behavior of $\mathcal{D}^{-1}=\mathcal{D}^{-1}(\omega)[64]$

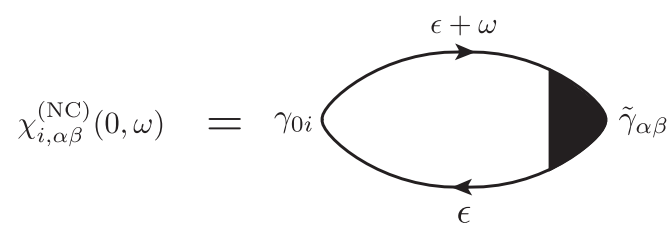

(b)

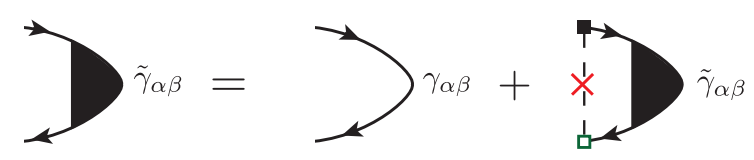

(c)

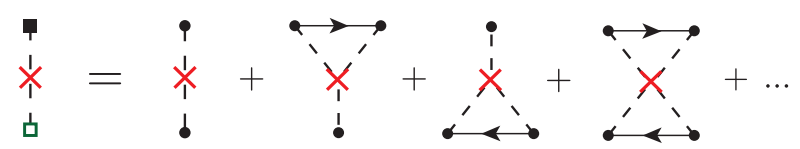

Figure 3. Diagrammatic technique for evaluation of generalized spin susceptibilities: (a) two-particle ladder diagram; (b) BS equation for the vertex renormalization; and (c) skeleton expansion of the ladder diagram in terms of an infinite series of two-particle, noncrossing diagrams. Full (open) square denotes a $T\left(T^{\dagger}\right)$ matrix insertion.

The formal result (Equation (30)) deserves a few comments. Firstly, $\mathcal{D}^{-1}$ spans in principle the entire Clifford algebra, which physically encodes the coupled dynamics of spin and other observables associated with the elements $\gamma_{\alpha \beta}$. However, by exploiting symmetries, $\mathcal{D}^{-1}$ can be reduced into block diagonal form, such that only some observables are coupled to the spin polarizations along the three spatial directions. Secondly, a distinct feature of Dirac systems is that spin densities are coupled to charge currents even in the case (considered here) of spatially uniform external perturbations $\mathbf{q}=0$. The linear Dirac dispersion of graphene is reflected in the form of the charge current $J_{i}=v \sigma_{i}$ and spin current $\mathcal{J}_{i}^{a}=v \sigma_{i} s_{a} / 2$ vertices, which do not depend explicitly on momentum; by virtue of that they can be directly identified (apart from constants) as elements of the Clifford algebra. Therefore, all the 
relevant information about coupling between currents and densities is built-in on the $16 \times 16$ diffusion operator (Equation (30)) in our formalism. This will allows us to obtain a unified description of spin relaxation processes and relativistic transport phenomena (e.g., charge-to-spin conversion) within our $\mathbf{q}=0$ formalism. We analyze the implications below.

The coupling of the electrons' spin to currents or other observables in the long wavelength limit also suggests two equivalent scenarios to study spin relaxation. The first natural choice is to consider spin injection and investigate the relaxation of the spin density profile (density-density response); alternatively, one can probe the spin response indirectly by exciting an observable coupled to the spin density through $\mathcal{D}^{-1}$. For instance, as we show in the following, one can drive a charge current via application of an electric field to obtain a in-plane spin polarization of carriers (ISGE). In that case, the information about the in-plane SRTs is readily accessible by examining how the steady state (Edelstein) polarization is achieved (density-current response).

Before moving on, let us stress that, within the Gaussian approximation, a useful relation can be derived connecting the generalized susceptibility Equation (25) and the renormalized vertex:

$$
\chi_{i, \alpha \beta}^{(\mathrm{NC})}(0, \omega)=\frac{\kappa}{2 \alpha} \sum_{\mu \nu} M_{\mu \nu 0 i}(\omega) \tilde{\gamma}_{\alpha \beta \mu \nu}(\omega)=\frac{\kappa}{2 \alpha}\left(\tilde{\gamma}_{\alpha \beta 0 i}(\omega)-\delta_{\alpha 0} \delta_{\beta i}\right)
$$

where $\alpha \equiv\left(2 \pi \tau N_{0}\right)^{-1}$ and we have used Equation (28). The above equation states the spin response can be solely obtained from the associated component of the renormalized vertex $\tilde{\gamma}_{\alpha \beta 0 i}$. A similar relation holds for other response functions. For example, the AC longitudinal (Drude) conductivity is written as

$$
\sigma_{x x}(\omega)=v^{2} \sum_{\mathbf{k}} \operatorname{tr}\left\{\gamma_{x 0} \mathcal{G}_{\mathbf{k}}^{R}(\epsilon+\omega) \tilde{\gamma}_{x 0}(\epsilon, \omega) \mathcal{G}_{\mathbf{k}}^{A}(\epsilon)\right\}=\frac{v^{2}}{\alpha}\left(\tilde{\gamma}_{x 0 x 0}(\omega)-1\right)
$$

Therefore, Equation (31) and similar relations allow identifying the components of a renormalized vertex with the associated observables, and will turn useful in the following.

Let us now determine the allowed couplings to $S_{x, y, z}$ by exploring symmetry. The model of Equation (1) is invariant under the group $C_{\infty v}$, which is an emergent symmetry of the continuum (long-wavelength) theory. As rotations in the continuum do not describe the sublattice symmetry $A \leftrightarrow B$ of the graphene system, a representation $U$ for the relevant set of discrete operations has to be considered. Relevant to us are $C_{2}$, the rotation of $\pi$ around the $\hat{z}$-axis exchanging sublattice (and valleys), and $R_{x}$, the reflection over the $\hat{x}$-axis. We have

$$
\begin{aligned}
U\left(C_{2}\right) & =\tau_{x} s_{z}, \\
U\left(R_{x}\right) & =\tau_{z} \sigma_{x} s_{y} r_{x} .
\end{aligned}
$$

where $r_{x}:(\mathbf{x}, \mathbf{y}) \rightarrow(\mathbf{x},-\mathbf{y})$ and $\tau_{i=x, y, z}$ are Pauli matrices acting on the valley degree of freedom. We also make use of isospin (valley) rotations $\Lambda_{x, y, z}[65,66]$

$$
\begin{aligned}
\Lambda_{x, y} & =\tau_{x, y} \sigma_{z}, \\
\Lambda_{z} & =\tau_{z} .
\end{aligned}
$$

For scalar disorder, it suffices to examine the form of the clean-system susceptibility at $\omega=0$

$$
\chi_{i, \alpha \beta}^{R A \text {,clean }} \equiv \frac{1}{4} \operatorname{Tr}\left[\gamma_{0 i} \mathcal{G}_{0}^{R}(\epsilon) \gamma_{\alpha \beta} \mathcal{G}_{0}^{A}(\epsilon)\right] .
$$


For any of the symmetries $\mathcal{S}$ listed above, we have $\mathcal{S}^{-1} G_{0}^{R / A} \mathcal{S}=\mathcal{G}_{0}^{R / A}$, and, inserting resolutions of the identity in the form $\mathcal{S}^{+} \mathcal{S}$ into Equation (37), we find

$$
\chi_{i, \alpha \beta}^{R A, \text { clean }}=\frac{p_{\alpha \beta} p_{0 i}}{4} \operatorname{Tr}\left[\gamma_{0 i} \mathcal{G}_{0}^{R}(\epsilon) \gamma_{\alpha \beta} \mathcal{G}_{0}^{A}(\epsilon)\right]=p_{\alpha \beta} p_{0 i} \chi_{i, \alpha \beta}^{R A, \text { clean }},
$$

where $p_{\alpha \beta}\left(p_{0 i}\right)= \pm 1$ is the parity of $\gamma_{\alpha \beta}\left(\gamma_{0 i}\right)$ under $\mathcal{S}$. From this result, we see that a nonzero response requires the operator $\gamma_{\alpha \beta}$ to have the same parity of the spin vertex under the action of any of $\mathcal{S}$. The allowed couplings and parities under $\mathcal{S}$ are shown in the Table 1 . As anticipated above, the in-plane components $S_{x(y)}$ are coupled to orthogonal charge currents $\sigma_{y(x)}$, as well as spin Hall currents $\gamma_{x z}\left(\gamma_{y z}\right)$ and staggered magnetizations $\gamma_{z y}\left(\gamma_{z x}\right)$ [43,44]. The out-of-plane component $S_{z}$ is instead coupled to a mass term $\sigma_{z}$ and in-plane spin currents $\gamma_{x x}, \gamma_{y y}$.

Table 1. Table summarizing the allowed couplings to the spin polarizations in the 2D Dirac-Rashba model with nonmagnetic scalar disorder.

\begin{tabular}{ccccc}
\hline Polarization & $\boldsymbol{C}_{\mathbf{2}}$ & $\boldsymbol{R}_{\boldsymbol{x}}$ & $\boldsymbol{\Lambda}_{\boldsymbol{x}, \boldsymbol{y}, \boldsymbol{z}}$ & Couplings \\
\hline$S_{x}$ & -1 & -1 & +1 & $\sigma_{y}, \gamma_{x z}, \gamma_{z y}$ \\
$S_{y}$ & -1 & +1 & +1 & $\sigma_{x}, \gamma_{y z}, \gamma_{z x}$ \\
$S_{z}$ & +1 & -1 & +1 & $\sigma_{z}, \gamma_{x x}, \gamma_{y y}$ \\
\hline
\end{tabular}

\subsection{Diffusive Equations and SRTs}

In the following, we choose to consider the in-plane spin response to an AC electric field $H_{\|}^{\text {ext }}=$ $-v \sigma_{i} A_{i}(\omega)=-(i \omega)^{-1} v \sigma_{i} \mathcal{E}_{i}(\omega), i=x, y$. This choice, as discussed above, is equivalent to consider in-plane spin injection, but has the advantage to allow for a unified description of spin dynamics and charge-spin interconversion, e.g., to capture the ISGE or similar effects $[40,67,68]$. For the out-of-plane spin dynamics, we take a spin-density perturbation $H_{\perp}^{\text {ext }}=\frac{1}{2} s_{z} B_{z}(\omega)$ (see Table 1).

\subsubsection{In-Plane Spin Dynamics}

Without loss of generality, let us consider the dynamics of the $\hat{y}$ polarization. According to Table 1 , $s_{y}$ is coupled to three operators: $\sigma_{x}, \sigma_{y} s_{z}$ and $\sigma_{z} s_{x}$. However, leading terms in the $(\epsilon \tau)^{-1}$ expansion are only contained in the $s_{y} / \sigma_{x}$ sub-block. Hence, to capture the SRTs, it suffices to restrict to this $2 \times 2$ algebra. As anticipated above, we consider here the response to an AC electric field $\mathcal{E}_{x}(\omega)$, associated with the vertex $\kappa \gamma_{x 0}=v \sigma_{x} \equiv v_{x}$. (Details of calculation and full form of the $4 \times 4$ diffusion operator is given in Appendices $C$ and D.) To capture purely diffusive processes, we expand $\mathcal{D}^{-1}(\omega)$ in the low-frequency and small SOC limits, $\omega \tau \ll 1$ and $\lambda \tau \ll 1$, respectively. In this regime, Equation (30) is written then as

$$
\left(\begin{array}{cc}
\frac{1}{2}(1-\imath \omega \tau) & \frac{\lambda}{\epsilon} \Gamma_{s}(1+3 \imath \omega \tau) \\
\frac{\lambda}{\epsilon} \Gamma_{s}(1+3 \imath \omega \tau) & \Gamma_{S}-\imath \omega \tau
\end{array}\right)\left(\begin{array}{c}
\tilde{\gamma}_{x 0 x 0} \\
\tilde{\gamma}_{x 00 y}
\end{array}\right)=\left(\begin{array}{l}
1 \\
0
\end{array}\right)
$$

where $\Gamma_{\mathrm{s}}=\tau / \tau_{\mathrm{s}}=2 \lambda^{2} \tau$. In the light of previous discussions (cf. Equations (31) and (32)), $\tilde{\gamma}_{x 0 x 0}$ and $\tilde{\gamma}_{x 00 y}$ are connected by a linear transformation to the steady-state charge current and the spin polarization (Appendix D).

Off-diagonal elements of Equation (39) carry in relation to diagonal ones an extra order of smallness $\lambda / \epsilon$, suggesting spin and charge to be weakly coupled in this limit. Their inclusion 
however encodes charge-to-spin interconversion and it is essential to get a correct physical description. The eigenvalues $-\imath \omega_{ \pm}$are found as

$$
\begin{aligned}
& -\imath \omega_{+} \simeq \frac{1}{\tau}\left(1+16 \frac{\Gamma_{s}^{3}}{\epsilon^{2} \tau}\right), \\
& -\imath \omega_{-} \simeq \frac{1}{\tau_{s}}\left(1-\frac{\Gamma_{s}^{3}}{\epsilon^{2} \tau}\right),
\end{aligned}
$$

and can be associated with charge current and spin relaxation times, respectively. We see then the SRT can be identified as the mass $(\omega=0)$ term of the spin-spin part of the diffusion

$$
\frac{1}{\tau_{s}} \equiv \frac{1}{\tau_{s}^{\|}} \simeq 1-M_{0 y 0 y}(\omega=0) \simeq 2 \lambda^{2} \tau
$$

Inverting Equation (39), we find

$$
\begin{aligned}
& \tilde{\gamma}_{x 0 x 0} \simeq \frac{1}{\tau} \frac{2}{-\imath \omega+\frac{1}{\tau}}, \\
& \tilde{\gamma}_{x 00 y} \simeq 2 \frac{\lambda}{\epsilon} \frac{1}{\tau} \frac{\Gamma_{s}}{-\imath \omega+\frac{\Gamma_{s}}{\tau}},
\end{aligned}
$$

from which, by using Equations (31) and (32), is it possible, upon Fourier transform, to derive the diffusive equation of motion for coupled charge-spin dynamics as

$$
\begin{aligned}
& \partial_{t} J_{x}(t)=-\frac{1}{2 \tau}\left(J_{x}(t)-J_{x}^{0}(t)\right), \\
& \partial_{t} S_{y}(t)=-\frac{1}{\tau_{s}^{\|}}\left(S_{y}(t)-S_{y}^{0}(t)\right),
\end{aligned}
$$

where $J_{x}^{0}(t) \equiv 2 v^{2} \mathcal{E}_{x}(t) / \alpha$ and $S_{y}^{0}(t) \equiv-\lambda \mathcal{E}_{x}(t) / \epsilon \alpha$. Note that charge current relaxation is regulated by the transport time $\tau_{\text {tr }} \equiv 2 \tau$, indicating the absence of backscattering $[43,54,55]$.

\subsubsection{Out-of-Plane Spin Dynamics}

For the out-of-plane spin dynamics we consider the renormalized vertex $\kappa \tilde{\gamma}_{0 z}=\frac{1}{2} \tilde{s}_{z}$. The off diagonal components of the associated $4 \times 4$ diffusion block contains sub-leading terms in the $(\epsilon \tau)^{-1}$ expansion (Appendix C), such that the out-of-plane SRTs can be calculated similarly to Equation (42) as

$$
\frac{1}{\tau_{s}^{\perp}} \simeq 1-M_{0 z 0 z}(\omega) \simeq 4 \lambda^{2} \tau
$$

The generalization of the equations of motion, i.e., Equations (45) and (46), in this case is written as

$$
\partial_{t} S_{z}(t)=-\frac{1}{\tau_{s}^{\perp}}\left(S_{z}(t)-S_{z}^{0}(t)\right)
$$

where $S_{z}^{0}(t)=\dot{B}_{z}(t) / 4 \alpha$ is the effect of the external perturbation (spin-injection field). The in-plane and out-of-plane SRTs are in the following relation

$$
\frac{1}{\tau_{s}^{\|}}=\frac{1}{2} \frac{1}{\tau_{s}^{\perp}}
$$

which is nothing but the well-known DP ratio for 2DEGs [61]. The above result has also been obtained for graphene within the time-dependent perturbation theory for the density matrix [42]. The agreement between graphene and the Rashba 2DEG results is expected at high electronic density $\epsilon \gg \lambda$. 


\subsection{SRT from the Conservation Laws in the DC Limit}

In this section, we demonstrate how the SRTs we have obtained above can be equivalently extracted in the static limit $\omega=0$. This remarkable result is rooted in the conservation laws associated to the disordered Dirac-Rashba Hamiltonian Equation (1) [43]. The first step is to write the Heisenberg equation of motion for the spin polarizations

$$
\partial_{t} S_{i}={ }_{\imath}\left[H, S_{i}\right]=\frac{2 \lambda}{v} \epsilon_{l j} \epsilon_{l i}^{c} J_{j}^{c}
$$

where $\epsilon_{l j}, \epsilon_{l i}^{c}$ are the second and third rank Levi-Civita tensors and $J_{j}^{c}=\left\langle\mathcal{J}_{j}^{c}\right\rangle$ is the $\hat{j}$-component of the pure spin current (with polarization " $c$ "). As before, we consider an electric field applied along the $\hat{x}$ direction. We find

$$
\partial_{t} S_{y}=\frac{2 \lambda}{v} J_{y}^{z}
$$

where $J_{y}^{z}$ is identified as the spin Hall current according to the chosen geometry. The spin Hall current is written in response to the electric field as

$$
J_{y}^{z}=\sigma_{y x}^{z} \mathcal{E}_{x}
$$

where $\sigma_{y x}^{z}$ is the DC spin Hall conductivity calculated according to Equation(25) with $\tilde{\gamma}_{0 y} \rightarrow v \tilde{\gamma}_{y z}$. As for now, no assumption has been made for the self-energy approximation associated to the scalar impurities field. Let us start from the more transparent Gaussian case. Using the corresponding version of Equation (31) for $\sigma_{y x}^{z}$, together with Equation (28) we have

$$
\sigma_{y x}^{z}=\frac{v^{2}}{2 \alpha} \tilde{\gamma}_{x 0 y z}=\frac{v^{2}}{2 \alpha}\left(M_{x 0 y z} \tilde{\gamma}_{x 0 x 0}+M_{0 y y z} \tilde{\gamma}_{x 00 y}\right) \text {. }
$$

In the latter, we have neglected the terms $M_{y z y z}$ and $M_{z x y z}$ which, as said above, provide higher order corrections in the $(\epsilon \tau)^{-1}$ expansion. Multiplying both sides of Equation (53) by the electric field $\mathcal{E}_{x}$, and using $S_{y}=\chi_{y, x 0} \mathcal{E}_{x}$ together with Equation (31), we find

$$
J_{y}^{z}=\frac{v^{2}}{2 \alpha} M_{x 0 y z} \tilde{\gamma} x 0 x 0 \mathcal{E}_{x}+v M_{0 y y z} S_{y}
$$

Despite the Dirac character of fermions, the steady-state case of the continuity equation Equation (51) imposes the latter spin Hall current to vanish, analogously to the 2DEG case [43]. This implies the establishment of the out-of-equilibrium value for the spin polarization as

$$
S_{y}^{0}=-\frac{\tilde{\gamma}_{x 0 x 0}}{2 \alpha v} \frac{M_{x 0 y z}}{M_{0 y y z}} \mathcal{E}_{x} .
$$

Evaluating the above quantities explicitly $\tilde{\gamma}_{x 0 x 0}=2, M_{x 0 y z} / M_{0 y y z}=\lambda / \epsilon$ and we recover the ISGE obtained in [44]. Using Equation (51), we finally arrive at

$$
J_{y}^{z}=v M_{0 y y z}\left(S_{y}-S_{y}^{0}\right)
$$

and therefore

$$
\partial_{t} S_{y} \equiv-\frac{1}{\tau_{s}^{\|}}\left(S_{y}-S_{y}^{0}\right),
$$

where we have identified the spin relaxation time

$$
\frac{1}{\tau_{s}^{\|}}=-2 \lambda M_{0 y y z}=2 \lambda^{2} \tau
$$


in perfect accordance with the result obtained above, Equation (42). The bubble $M_{0 y y z}$ is therefore what completely determines the in-plane spin relaxation.

We now ask how the above result is modified when treating the self-energy in the T-matrix approximation. The Bethe Salpeter equation Equation (26) now reads

$$
\tilde{\gamma}_{x 0}(\epsilon)=\gamma_{x 0}+n_{i} \sum_{\mathbf{k}} T^{R}(\epsilon) \mathcal{G}_{\mathbf{k}}^{R}(\epsilon) \tilde{\gamma}_{x 0}(\epsilon) \mathcal{G}_{\mathbf{k}}^{A}(\epsilon) T^{A}(\epsilon),
$$

where $T^{R / A}(\epsilon)$ is the single-impurity $T$-matrix in the $\mathrm{R} / \mathrm{A}$ sectors introduced in Equation (8). Projecting onto the Clifford algebra, similar to Equation (28), we have

$$
\tilde{\gamma}_{x 0 \varrho \varsigma}=\delta_{x \varrho} \delta_{0 \varsigma}+\sum_{\mu \nu \zeta \xi=0, x, y, z} Y_{\varrho \zeta \zeta \xi} N_{\mu \nu \zeta \xi} \tilde{\gamma}_{x 0 \mu \nu}
$$

where we have defined

$$
\begin{aligned}
N_{\mu \nu \zeta \zeta} & =\frac{n_{i}}{4} \sum_{\mathbf{k}} \operatorname{tr}\left(\mathcal{G}_{\mathbf{k}}^{R} \gamma_{\mu \nu} \mathcal{G}_{\mathbf{k}}^{A} \gamma_{\zeta \zeta}\right), \\
Y_{\varrho \zeta \zeta \zeta} & =\frac{1}{4} \operatorname{tr}\left[T^{A} \gamma_{\varrho \zeta} T^{R} \gamma_{\zeta \zeta}\right] .
\end{aligned}
$$

Recasting Equation (60) in vector notation, in the same spirit of Equation (30), we have

$$
\tilde{\gamma}_{x 0}=\gamma_{x 0}+Y N^{\mathrm{t}} \tilde{\gamma}_{x 0}
$$

and consequently

$$
\gamma^{-1}\left(\tilde{\gamma}_{x 0}-\gamma_{x 0}\right)=N^{t} \tilde{\gamma}_{x 0}
$$

The latter equation allows again to find a connection with the observables. For example, the generalization of Equation (31) is written as

$$
\chi_{y, x 0}=\frac{2 v}{n_{i}} \sum_{\mu v} N_{\mu v 0 y} \tilde{\gamma}_{x 0 \mu v}=\frac{2 v}{n_{i}} \sum_{\mu v} Y_{0 y \mu v}^{-1} \tilde{\gamma}_{x 0 \mu v} .
$$

The spin Hall conductivity instead is found as

$$
\sigma_{y x}^{z}=\frac{2 v^{2}}{n_{i}} \sum_{\mu \nu} Y_{y z \mu \nu}^{-1} \tilde{\gamma}_{x 0 \mu \nu}
$$

Different from the Gaussian case, where we could relate the response of an observable uniquely to the associated component of the renormalized vertex, in the $T$-matrix limit, in principle, all components of $\tilde{\gamma}_{x 0}$ would contribute, each of them with weight given by $Y^{-1}$. In the limiting case of unitary limit $u_{0} \rightarrow \infty$, where $\lim _{u_{0} \rightarrow \infty} T^{R / A}=-\frac{1}{g_{0}^{R / A}}$, we find a simplification as

$$
Y_{\varrho \zeta \zeta \zeta}^{-1}=\left|g_{0,0}^{R}\right|^{2} \delta_{\varrho \zeta} \delta_{\zeta \zeta} .
$$

This implies that, for Equation(66), a relation similar to the Gaussian case is obtained

$$
J_{y}^{z}=\sigma_{y x}^{z} \mathcal{E}_{x}=\frac{2 v^{2}}{n_{i}}\left|g_{0,0}^{R}\right|^{2} \tilde{\gamma}_{x 0 y z} \mathcal{E}_{x}=\frac{2 v^{2}}{n_{i}} N_{x 0 y z} \tilde{\gamma}_{x 0 x 0} \mathcal{E}_{x}+v N_{00 y z}\left|g_{0,0}^{R}\right|^{-2} S_{y},
$$


where we have restricted ourselves again to the dominant subspace $\sigma_{x} / s_{y}$. After standard algebra, we arrive at

$$
\partial_{t} S_{y}=\frac{2 \lambda}{v} \sigma_{y x}^{z} \mathcal{E}_{x}=\frac{2 \lambda}{v} v N_{0 y y z}\left(S_{y}-S_{y}^{0}\right)
$$

and the SRT defined as

$$
\frac{1}{\tau_{s}^{\|}}=2 \lambda\left|g_{0,0}^{R}\right|^{-2} N_{00 y z}=2 \lambda \frac{1}{\epsilon^{2}} \frac{16 \pi^{2} v^{4}}{\pi^{2}+\mathcal{L}_{\mathrm{II}}^{2}} N_{00 y z}=2 \lambda^{2} \tau,
$$

where we have used the definition of $\tau$ in the unitary limit, Equation(20). We conclude that the the formal expression connecting $\tau_{s}$ and $\tau$ (the DP relation) is the same as found in the Gaussian limit for the self-energy. However, given the different dependence of $\tau$ on the Fermi level in the two approximations—cf. Equations (18) and (20)—one has

$$
\frac{\tau(\epsilon)}{\tau_{s}^{\|}(\epsilon)}= \begin{cases}\frac{2 \lambda^{2}}{\epsilon^{2}}\left(\frac{2 v^{2}}{n_{i} u_{0}^{2}}\right)^{2} & \text { Gaussian, } \\ \epsilon^{2} \frac{\lambda^{2}}{2}\left(\frac{\pi^{2}+\mathcal{L}_{\Pi I}^{2}}{4 \pi^{2} n_{i} v^{2}}\right)^{2} & \text { Unitary. }\end{cases}
$$

The SRT associated to the out-of-plane component can be derived along the same lines. The relevant Heisenberg equation now reads

$$
\partial_{t} S_{z}=-\frac{2 \lambda}{v}\left(J_{x}^{x}+J_{y}^{y}\right)
$$

and a similar reasoning that led to Equation (58) allows us to conclude

$$
\frac{1}{\tau_{S}^{\perp}}=2 \lambda\left(M_{0 z x x}+M_{0 z y y}\right)=4 \lambda^{2} \tau,
$$

in the Gaussian limit, and a similar relation for the unitary limit.

\subsection{Discussion}

Here, we discuss the DP relation obtained in Equation (71) within the Gaussian and unitary limits of potential scattering. The energy dependence of the spin lifetime for fixed impurity concentration is shown in Figure 4. Away from the Dirac point, within the Gaussian approximation, the spin lifetime increases linearly since $\tau \propto \epsilon^{-1}$ (see Equation (18)). In the unitary limit, instead, one has a linear dependence $\tau \propto \epsilon$ (see Equation (20)), leading to vanishing spin lifetime at high electron doping. On the other hand, near the Dirac point, the noncrossing approximation breaks down. It is not surprising that the spin lifetime dependences are found to be nonphysical as $\epsilon \rightarrow 0$ : vanishingly small for the Gaussian limit and divergent for the unitary limit. To overcome this limitation, one needs to evaluate crossing diagrams encoding quantum coherent processes, which includes weak localization corrections and diffractive skew-scattering from two or more impurities $[54,69,70]$. However, here, we are mostly interested in the diffusive regime away from the Dirac point $\epsilon \tau \gg 1$, thus neglecting interference effects that can correct the standard DP relation [32,33,71]. However, an important refinement is possible within the noncrossing formalism used here by evaluating the $O\left(n_{i}^{2}\right)$ terms in Equation (8). Such higher-order terms encode the strong renormalization of the single-particle propagators by incoherent multiple scattering approaching the Dirac point. To show this, it suffices to resume the 
infinite class of "rainbow" diagrams, a scheme known as self-consistent Born approximation (SCBA). The SCBA self-energy is given by the solution of the following self-consistent equation [66]

$$
\left.\frac{1}{2 \tau}\right|_{\mathrm{SCBA}}=-\Im \Sigma_{\mathrm{SCBA}}(\epsilon)=-\Im\left[\frac{n_{i}}{4 \pi v^{2}}\left(\epsilon-\Sigma_{\mathrm{SCBA}}(\epsilon)\right) \log \left(\frac{-\Lambda^{2}}{\left(\epsilon-\Sigma_{\mathrm{SCBA}}(\epsilon)\right)^{2}}\right)\right] .
$$

In Figure 4, we show that the SCBA provides a physical (finite, nonzero) $\tau_{s}$ approaching the Dirac point. To obtain a representative curve, we take $\lambda=1 \mathrm{meV}$ and we choose the impurity density and the scattering strength such that the SCBA nonperturbative energy scale $\Gamma=\Lambda e^{-2 \pi v^{2} /\left(n_{i} u_{0}^{2}\right)}[66]$ is a few tens meV. The in-plane SRT is then found to lie in the range 50-100 ps. Concerning the magnitude of $\tau_{s}^{\|}$we note the result is compatible with previous reports where the (uniform or random) Rashba SOC is treated by semiclassical or numerical approaches [32,42].

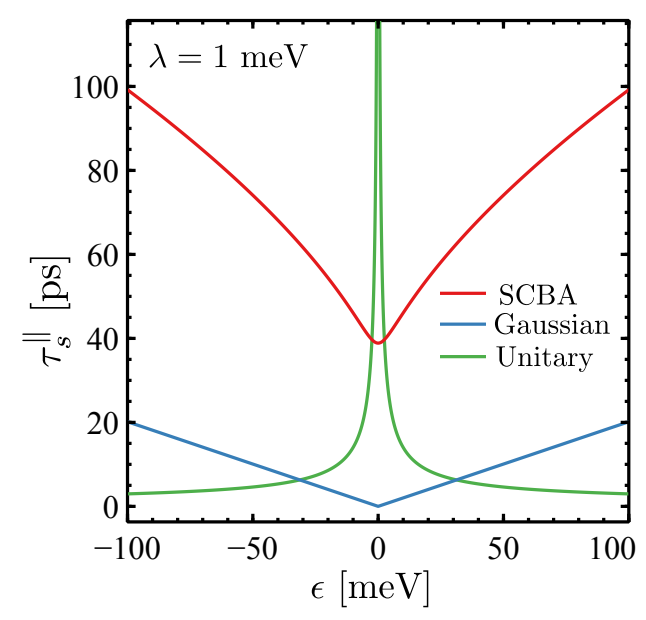

Figure 4. DP in-plane spin relaxation time calculated according different schemes for the self-energy: SCBA (red line), Gaussian (blue line) and unitary limit (green line). The most important feature obtained within the SCBA is a strong renormalization of $\tau_{s}^{\|}$in the vicinity of the Dirac point, reflecting a disorder-induced finite density of states in that region. In the plot, $\lambda=1 \mathrm{meV}$ and $\Gamma=60 \mathrm{meV}$.

\section{Conclusions}

In the present work, we laid the foundations of a general microscopic theory of diffusive transport and spin relaxation in 2D Dirac systems subject to spin-orbit interactions. Our work represents the logical extension of the previously-developed diagrammatic treatments $[61,62]$ to all orders in the scattering potential, for disordered electron systems with an enlarged pseudospin $\otimes$ spin Clifford algebra $[43,44]$. We applied the formalism to the paradigmatic case of 2D Dirac fermions with Rashba spin-orbit coupling considering the purely diffusive regime $\lambda \tau \ll 1 \ll \epsilon \tau$. We demonstrated how the Dyakonov-Perel relation between momentum and spin lifetime $\tau \propto \tau_{s}^{-1}$ holds in both the Gaussian (weak short-range scatterers) and the unitary (strong short-range scatterers) limits, despite the drastic different dependence momentum scattering times $\tau=\tau(\epsilon)$ in the two regimes. We derived the same result both by direct diagrammatic resummation (in the noncrossing approximation) and by exploiting the conservation laws of the theory in the zero-frequency limit. Under the diffusive regime $\lambda \tau \ll 1 \ll \epsilon \tau$ is not possible to study the dynamics in the region of Fermi energies comparable to the Rashba pseudogap region $\epsilon \sim 2 \lambda$, which was recently predicted to display interesting out-of-equilibrium phenomena [44]. The strong spin-momentum locking approaching this regime lets us infer a modification of the relation between $\tau_{s}$ and $\tau$ towards the Elliot-Yafet type $\tau_{s} \propto \tau$. Our theory sets the stage to study the spin dynamics in that regime. This topic has become of renewed great interest due to recent progresses in graphene-based heterostructures, where the spin relaxation anisotropy has been recognized as a viable tool to estimate the induced large spin-orbital effects [72-75]. 
Author Contributions: The authors equally contributed to this work.

Funding: M.O. and A.F. acknowledge funding from EPSRC (Grant No. EP/N004817/1).

Acknowledgments: The authors are grateful to Ignacio Wilson-Rae for stimulating discussions.

Conflicts of Interest: The authors declare no conflict of interest.

\section{Appendix A. Clean Green's Function}

The explicit form of the clean single-particle Green's function is

$$
\mathcal{G}_{0 \mathbf{k}}^{a}(\epsilon)=-\frac{1}{2} \sum_{\mu= \pm 1} L_{0 \mu}^{a}\left[(\lambda+\mu \epsilon) \gamma_{0}+v \sigma \cdot \mathbf{k}-\frac{\mu \epsilon}{2} \gamma_{r}+v(\mathbf{s} \times \mathbf{k})_{z}+\lambda \gamma_{z z}+\delta M_{2 \phi_{\mathbf{k}}}\right]
$$

where

$$
\begin{gathered}
L_{0 \mu}^{A(R)}=\frac{\mu}{v^{2} k^{2}-\epsilon^{2}-2 \mu \lambda \epsilon \pm \imath 0^{+} \operatorname{sign}(\epsilon-\mu \lambda)}, \\
\delta M_{2 \phi_{\mathbf{k}}}=-\frac{1}{2}(\epsilon+2 \mu \lambda)\left[\left(\sigma_{y} s_{y}-\sigma_{x} s_{x}\right) \sin 2 \phi_{\mathbf{k}}+\left(\sigma_{x} s_{y}+\sigma_{y} s_{x}\right) \cos 2 \phi_{\mathbf{k}}\right],
\end{gathered}
$$

and $\phi_{\mathbf{k}}$ is the angle formed by the wavevector with $\hat{k}_{x}$.

\section{Appendix B. Integrals and Expansion}

The current work makes extensive use of momentum integrations involving products of two renormalized Green's functions with analyticity opposite halves of the complex plane (see e.g., Equations (24) and (26)). The retarded function is displaced in energy by the amount $\omega$. Similar to the bare Green's function decomposition Equations (A1) and (A3), we write the renormalized (disorder averaged) propagators as

$$
\mathcal{G}_{\mathbf{k}}^{a}(\epsilon)=M_{1 \mathbf{k}}^{a}(\epsilon) L_{1 \mathbf{k}}^{a}(\epsilon)+M_{2 \mathbf{k}}^{a}(\epsilon) L_{2 \mathbf{k}}^{a}(\epsilon),
$$

where $M_{i \mathbf{k}}^{a}(\epsilon)=M_{i}^{a(0)}+v^{2} k^{2} M_{i}^{a(2)}, i=\{1,2\}$ are matrix coefficients and the kernels $L_{\mu}^{a}=L_{i \mathbf{k}}^{a}$ are obtained in the Gaussian limit from the functions $L_{0 \mu}$ of Equation(A2) by analytical continuation $\epsilon \rightarrow \epsilon+a \frac{l}{2 \tau}$. In the $T$ matrix approach, the analytical continuation has to be performed as to include the other matrix structure of the self-energy $\propto \gamma_{r}, \gamma_{z z}$ [44]. We can generically write

$$
L_{i \mathbf{k}}^{a}(\epsilon)=\frac{1}{v^{2} k^{2}-z_{i}^{a}(\epsilon)}
$$

where $z_{i}^{a}(\epsilon)$ are complex quantities. Given the decomposition in Equation (A4), the integrals we need to solve are reduced to product of two kernels in different combinations, accompanied or not by a factor $v^{2} k^{2}$. Terms proportional to $v^{4} k^{4}$ can be shown to vanish upon angular integration $\int d \phi_{\mathbf{k}}$. We write below an exact solution and then expand at linear order in $\omega$. For simplicity, we show the results for the Gaussian approximation. The first type of integrals is

$$
\begin{aligned}
\Gamma_{i j}=\int_{0}^{\infty} \frac{d k k}{2 \pi} L_{i \mathbf{k}}^{R}(\epsilon+\omega) L_{j \mathbf{k}}^{A}(\epsilon) & =\int_{0}^{\infty} \frac{d k k}{2 \pi} \frac{1}{v^{2} k^{2}-z_{i \mathbf{k}}^{R}(\epsilon+\omega)} \frac{1}{v^{2} k^{2}-z_{j \mathbf{k}}^{A}(\epsilon)} \\
= & \frac{1}{z_{i \mathbf{k}}^{R}(\epsilon+\omega)-z_{j \mathbf{k}}^{A}(\epsilon)} \int_{0}^{\infty} \frac{d k k}{2 \pi}\left(\frac{1}{v^{2} k^{2}-z_{i \mathbf{k}}^{R}(\epsilon+\omega)}-\frac{1}{v^{2} k^{2}-z_{j \mathbf{k}}^{A}(\epsilon)}\right) \\
= & \frac{1}{4 \pi v^{2}} \frac{1}{z_{i \mathbf{k}}^{R}(\epsilon+\omega)-z_{j \mathbf{k}}^{A}(\epsilon)} \times \\
& {\left[-\log \left(-z_{i \mathbf{k}}^{R}(\epsilon+\omega)\right)+\log \left(-z_{j \mathbf{k}}^{A}(\epsilon)\right)-\left(\frac{1}{z_{i \mathbf{k}}^{R}(\epsilon)} \partial_{\epsilon} z_{i \mathbf{k}}^{R}(\epsilon)\right) \omega\right], }
\end{aligned}
$$


where the principal branch of the log function has been chosen. Note $z_{1}^{a}(\lambda) \rightarrow z_{2}^{a}(-\lambda)$. Thus, $\Gamma_{11}(\lambda)=\Gamma_{22}(-\lambda)$ and $\Gamma_{12}(\lambda)=\Gamma_{21}(-\lambda)$. At linear order in $\omega$, we find

$$
\begin{gathered}
\Gamma_{11}=\frac{1}{4 \pi v^{2}}\left[\frac{\pi}{\epsilon+\lambda}-\frac{1}{\epsilon(\epsilon+2 \lambda)}+\imath \pi \omega \tau \frac{\tau}{\epsilon+\lambda}\right], \\
\Gamma_{22}=\Gamma_{11}(\lambda \rightarrow-\lambda), \\
\Gamma_{12}=\frac{1}{4 \pi v^{2}}\left[\frac{2 \imath \pi}{4 \epsilon \lambda}-\omega \frac{\epsilon+\lambda}{2 \epsilon^{2} \lambda(\epsilon+2 \lambda)}\left(1+\imath \pi \frac{\epsilon+2 \lambda}{2 \lambda}\right)\right], \\
\Gamma_{21}=\Gamma_{12}(\lambda \rightarrow-\lambda),
\end{gathered}
$$

where we have retained leading order terms in $(\epsilon \tau)^{-1}$. The other class of integrals we need to solve is

$$
\begin{aligned}
\Gamma_{i j}^{(3)} & =\int_{0}^{\Lambda / v} \frac{d k k^{3}}{2 \pi} L_{i \mathbf{k}}^{R}(\epsilon+\omega) L_{j \mathbf{k}}^{A}(\epsilon)=\int_{0}^{\Lambda / v} \frac{d k k^{3}}{2 \pi} \frac{1}{v^{2} k^{2}-z_{i \mathbf{k}}^{R}(\epsilon+\omega)} \frac{1}{v^{2} k^{2}-z_{j \mathbf{k}}^{A}(\epsilon)} \\
& =\frac{1}{4 \pi v^{2}} \frac{z_{i \mathbf{k}}^{R}(\epsilon+\omega)-z_{j \mathbf{k}}^{A}(\epsilon)}{2 \imath}\left[\operatorname{Im}\left(z_{i \mathbf{k}}^{R}(\epsilon) \log \left(\frac{\Lambda^{2}}{-z_{i \mathbf{k}}^{R}(\epsilon)}\right)\right)-\partial_{\epsilon} z_{i \mathbf{k}}^{R}(\epsilon)\left(1-\log \left(\frac{\Lambda^{2}}{-z_{i \mathbf{k}}^{R}(\epsilon)}\right)\right) \omega\right],
\end{aligned}
$$

where the ultraviolet cutoff $\Lambda / v \gg k_{\mathrm{F}}$ has been introduced to regularize the integrals. A careful evaluation yields

$$
\begin{aligned}
& \Gamma_{11}^{(3)}=\frac{1}{4 \pi v^{2}}\left[\frac{\pi \epsilon(\epsilon+2 \lambda) \tau}{\epsilon+\lambda}(1+\imath \omega \tau)+\log \left|\frac{\Lambda^{2}}{\epsilon^{2}+2 \epsilon \lambda}\right|-1-\omega \frac{\pi \epsilon \lambda \tau}{2(\epsilon+\lambda)^{2}}\right], \\
& \Gamma_{12}^{(3)}=\frac{1}{4 \pi v^{2}}\left[\frac{2 \imath \pi \epsilon+2 \lambda \mathcal{L}_{\mathrm{II}}}{4 \lambda}-\omega \frac{\epsilon+\lambda}{2 \epsilon \lambda}\left(1+2 \pi \frac{\epsilon+2 \lambda}{4 \lambda}\right)\right],
\end{aligned}
$$

and the expressions for $1 \leftrightarrow 2$ are again obtainable with the replacement $\lambda \rightarrow-\lambda$.

\section{Appendix C. Full Form of the Diffusion}

Here, we report the full form of the two relevant blocks of the diffusion, involving $S_{y, z}$. The expressions are provided at leading order in the expansions for $\omega \tau \ll \lambda \tau \ll 1 \ll \epsilon \tau$.

- $\quad$ Subspace $\sigma_{x}, s_{y}, \sigma_{y} s_{z}, \sigma_{z} s_{x}$

$$
\left.\mathcal{D}^{-1}\right|_{s_{y}}=\left(\begin{array}{cccc}
\frac{1}{2}(1-\imath \omega \tau) & \frac{2 \lambda^{3} \tau^{2}}{\epsilon}(1+3 \imath \omega \tau) & -\frac{\lambda^{2} \tau}{\epsilon}(1+2 \imath \omega \tau) & \frac{\lambda^{3} \tau}{\epsilon^{2}}(1+2 \imath \omega \tau) \\
\frac{2 \lambda^{3} \tau^{2}}{\epsilon}(1+3 \imath \omega \tau) & 2 \lambda^{2} \tau^{2}-\imath \omega \tau & -\lambda \tau(1+2 \imath \omega \tau) & \frac{\lambda^{2} \tau}{\epsilon}(1+2 \imath \omega \tau) \\
\frac{\lambda^{2} \tau}{\epsilon}(1+2 \imath \omega \tau) & \lambda \tau(1+2 \imath \omega \tau) & \frac{1}{2}(1-\imath \omega \tau) & \frac{\lambda}{2 \epsilon}(1+\imath \omega \tau) \\
-\frac{\lambda^{3} \tau}{\epsilon^{2}}(1+2 \imath \omega \tau) & -\frac{\lambda^{2} \tau}{\epsilon}(1+2 \imath \omega \tau) & \frac{\lambda}{2 \epsilon}(1+\imath \omega \tau) & 1-\imath \omega \tau \frac{\lambda^{2}}{2 \epsilon^{2}}
\end{array}\right),
$$

- $\quad$ Subspace $s_{z}, \sigma_{x} s_{x}, \sigma_{y} s_{y}, \sigma_{z}$

$$
\left.\mathcal{D}^{-1}\right|_{s_{z}}=\left(\begin{array}{cccc}
4 \lambda^{2} \tau^{2}-\imath \omega \tau & \lambda \tau(1+2 \imath \omega \tau) & \lambda \tau(1+2 \imath \omega \tau) & -\frac{\lambda}{\pi \tau \epsilon^{2}}+O\left[(\epsilon \tau)^{-4}\right] \\
-\lambda \tau(1+2 \imath \omega \tau) & \frac{1}{2}(1-\imath \omega \tau) & \frac{\lambda^{2} \tau^{2}}{2}(1+3 \imath \omega \tau) & O\left[(\epsilon \tau)^{-4}\right] \\
-\lambda \tau(1+2 \imath \omega \tau) & \frac{\lambda^{2} \tau^{2}}{2}(1+3 \imath \omega \tau) & \frac{1}{2}(1-\imath \omega \tau) & O\left[(\epsilon \tau)^{-4}\right] \\
-\frac{\lambda}{\pi \tau \epsilon^{2}}+O\left[(\epsilon \tau)^{-4}\right] & O\left[(\epsilon \tau)^{-4}\right] & O\left[(\epsilon \tau)^{-4}\right] & 1+\frac{\imath \omega}{4 \epsilon^{2} \tau}
\end{array}\right) .
$$




\section{Appendix D. Equations for Observables Instead of Vertices}

In the main text, we have written equations of motion for the renormalized vertices, rather than for the observables themselves. As an example, here, we report the diffusive matrix $\mathcal{D}^{-1}$ for the observables, in the relevant sub-block $s_{y} / \sigma_{x}$ for the in-plane spin dynamics. In addition, here, we consider the response to an external electric field $\mathcal{E}_{x}$. To this aim, we recall in the Gaussian approximation (cf. Equations (31) and (32))

$$
\begin{aligned}
& J_{x}=\sigma_{x x} \mathcal{E}_{x}=\frac{v^{2}}{\alpha}\left(\tilde{\gamma}_{x 0 x 0}-1\right) \mathcal{E}_{x}, \\
& S_{y}=v \chi_{y, 0 x} \mathcal{E}_{x}=\frac{v}{2 \alpha} \tilde{\gamma}_{x 00 y} \mathcal{E}_{x} .
\end{aligned}
$$

Manipulating Equation(30), we have

$$
\mathcal{D}^{-1} \tilde{\gamma}_{x 0}=\gamma_{x 0} \Longrightarrow \mathcal{C} \tilde{\gamma}_{x 0}=\mathcal{C D} \gamma_{x 0}
$$

where we have defined the matrix

$$
\mathcal{C}=\frac{v \mathcal{E}_{x}}{\alpha} \operatorname{diag}\left(v, \frac{1}{2}\right) .
$$

Consequently, by subtracting from both sides $v^{2} \mathcal{E}_{x} \gamma_{x 0} / \alpha$, we have

$$
\frac{v \mathcal{E}_{x}}{\alpha}\left[\left(\begin{array}{c}
v \tilde{\gamma}_{x 0 x 0} \\
\frac{\tilde{\gamma}_{x 0 x 0}}{2}
\end{array}\right)-\left(\begin{array}{c}
v \\
0
\end{array}\right)\right] \equiv\left(\begin{array}{c}
J_{x} \\
S_{y}
\end{array}\right)=\left(\mathcal{C D}-\frac{v^{2}}{\alpha} \mathcal{E}_{x} \gamma_{0}\right) \gamma_{x 0} .
$$

We conclude the diffusive matrix for the observables is

$$
\mathcal{D}_{\text {obs }}^{-1}=\left(\mathcal{C D}-\frac{v^{2}}{\alpha} \mathcal{E}_{x} \gamma_{0}\right)^{-1} .
$$

Direct inspection shows that $\mathcal{D}_{\text {obs }}^{-1}$ and $\mathcal{D}^{-1}$ share the same poles structures, justifying the approach in the main text.

\section{References and Notes}

1. Huertas-Hernando, D.; Guinea, F.; Brataas, A. Spin-orbit coupling in curved graphene, fullerenes, nanotubes, and nanotube caps. Phys. Rev. B 2006, 74, 155426. [CrossRef]

2. Konschuh, S.; Gmitra, M.; Fabian, J. Tight-binding theory of the spin-orbit coupling in graphene. Phys. Rev. B 2010, 82, 245412. [CrossRef]

3. Pesin, D.; MacDonald, A.H. Spintronics and pseudospintronics in graphene and topological insulators. Nat. Mater. 2012, 11, 409-416. [CrossRef] [PubMed]

4. Han, W.; Kawakami, R.; Gmitra, M.; Fabian, J. Graphene spintronics. Nat. Nanotechnol. 2014, 9, 794-807. [CrossRef] [PubMed]

5. Roche, S.; Valenzuela, S.O. Graphene spintronics: Puzzling controversies and challenges for spin manipulation. J. Phys. D Appl. Phys. 2014, 47, 094011. [CrossRef]

6. Lara-Avila, S.; Kubatkin, S.; Kashuba, O.; Folk, J.A.; Lüscher, S.; Yakimova, R.; Janssen, T.; Tzalenchuk, A.; Fal'ko, V. Influence of impurity spin dynamics on quantum transport in epitaxial graphene. Phys. Rev. Lett. 2015, 115, 106602. [CrossRef] [PubMed]

7. Lundeberg, M.B.; Yang, R.; Renard, J.; Folk, J.A. Defect-mediated spin relaxation and dephasing in graphene. Phys. Rev. Lett. 2013, 110, 156601. [CrossRef] [PubMed]

8. Raes, B.; Scheerder, J.E.; Costache, M.V.; Bonell, F.; Sierra, J.F.; Cuppens, J.; van de Vondel, J.; Valenzuela, S.O. Determination of the spin-lifetime anisotropy in graphene using oblique spin precession. Nat. Commun. 2016, 7, 11444. [CrossRef] [PubMed] 
9. Omar, S.; Guimares, M.H.D.; Kaverzin, A.; van Wees, B.J.; Vera-Marun, I.J. Spin relaxation 1/f noise in graphene. Phys. Rev. B 2017, 95, 081403(R) . [CrossRef]

10. Johnson, M.; Silsbee, R.H. Interfacial charge-spin coupling: Injection and detection of spin magnetization in metals. Phys. Rev. Lett. 1985, 55, 1790. [CrossRef] [PubMed]

11. Jedema, F.J.; Filip, A.T.; van Wees, B.J. Electrical spin injection and accumulation at room temperature in an all-metal mesoscopic spin valve. Nature 2001, 410, 345. [CrossRef] [PubMed]

12. Tombros, N.; Jozsa, C.; Popinciuc, M.; Jonkman, H.T.; van Wees, B.J. Electronic spin transport and spin precession in single graphene layers at room temperature. Nature 2007, 448, 571-574. [CrossRef] [PubMed]

13. Józsa, C.; Maassen, T.; Popinciuc, M.; Zomer, P.J.; Veligura, A.; Jonkman, H.T.; van Wees, B.J. Linear scaling between momentum and spin scattering in graphene. Phys. Rev. B 2009, 80, 241403(R). [CrossRef]

14. Popinciuc, M.; Józsa, C.; Zomer, P.J.; Tombros, N.; Veligura, A.; Jonkman, H.T.; Van Wees, B.J. Electronic spin transport in graphene field-effect transistors. Phys. Rev. B 2009, 80, 214427. [CrossRef]

15. Han, W.; Pi, K.; McCreary, K.M.; Li, Y.; Wong, J.J.; Swartz, A.G.; Kawakami, R.K. Tunneling spin injection into single layer graphene. Phys. Rev. Lett. 2010, 105, 167202. [CrossRef] [PubMed]

16. Yang, T.-Y.; Balakrishnan, J.; Volmer, F.; Avsar, A.; Jaiswal, M.; Samm, J.; Ali, S.R.; Pachoud, A.; Zeng, M.; Popinciuc, M.; et al. Observation of long spin-relaxation times in bilayer graphene at room temperature. Phys. Rev. Lett. 2011, 107, 047206. [CrossRef] [PubMed]

17. Han, W.; Kawakami, R. Spin relaxation in single-layer and bilayer graphene. Phys. Rev. Lett. 2011, 107, 047207. [CrossRef] [PubMed]

18. Pi, K.; Han, W.; McCreary, K.M.; Swartz, A.G.; Li, Y.; Kawakami, R.K. Manipulation of spin transport in graphene by surface chemical doping. Phys. Rev. Lett. 2010, 104, 187201. [CrossRef] [PubMed]

19. Jo, S.; Ki, D.K.; Jeong, D.; Lee, H.J.; Kettemann, S. Spin relaxation properties in graphene due to its linear dispersion. Phys. Rev. B 2011, 84, 075453. [CrossRef]

20. Zomer, P.J.; Guimarães, M.H.D.; Tombros, N.; van Wees, B.J. Long-distance spin transport in high-mobility graphene on hexagonal boron nitride. Phys. Rev. B 2012, 86, 161416. [CrossRef]

21. Drögeler, M.; Volmer, F.; Wolter, M.; Terrés, B.; Watanabe, K.; Taniguchi, T.; Güntherodt, G.; Stampfer, C.; Beschoten, B. Nanosecond Spin Lifetimes in Single- and Few-Layer Graphene-hBN Heterostructures at Room Temperature. Nano Lett. 2014, 14, 6050-6055. [CrossRef] [PubMed]

22. Drögeler, M.; Franzen, C.; Volmer, F.; Pohlmann, T.; Banszerus, L.; Wolter, M.; Watanabe, K.; Taniguchi, T.; Stampfer, C.; Beschoten, B. Spin lifetimes exceeding $12 \mathrm{~ns}$ in graphene nonlocal spin valve devices. Nano Lett. 2016, 16, 3533-3539. [CrossRef] [PubMed]

23. Neto, A.H.C.; Guinea, F. Impurity-induced spin-orbit coupling in graphene. Phys. Rev. Lett. 2009, 103, 026804. [CrossRef] [PubMed]

24. Huertas-Hernando, D.; Guinea, F.; Brataas, A. Spin-Orbit-Mediated Spin Relaxation in Graphene. Phys. Rev. Lett. 2009, 103, 146801. [CrossRef] [PubMed]

25. Ertler, C.; Konschuh, S.; Gmitra, M.; Fabian, J. Electron spin relaxation in graphene: The role of the substrate. Phys. Rev. B 2009, 80, 041405(R). [CrossRef]

26. Kochan, D.; Gmitra, M.; Fabian, J. Spin relaxation mechanism in graphene: Resonant scattering by magnetic impurities. Phys. Rev. Lett. 2014, 112, 116602. [CrossRef] [PubMed]

27. Maassen, T.; Dejene, F.K.; Guimaraes, M.H.D.; Józsa, C.; Van Wees, B.J. Comparison between charge and spin transport in few-layer graphene. Phys. Rev. B 2011, 83, 115410. [CrossRef]

28. Volmer, F.; Drögeler, M.; Maynicke, E.; von den Driesch, N.; Boschen, M.L.; Güntherodt, G.; Beschoten, B. Role of $\mathrm{MgO}$ barriers for spin and charge transport in $\mathrm{Co} / \mathrm{MgO} /$ graphene nonlocal spin-valve devices. Phys. Rev. B 2013, 88, 161405(R) . [CrossRef]

29. Fedorov, D.V.; Gradhand, M.; Ostanin, S.; Maznichenko, I.V.; Ernst, A.; Fabian, J.; Mertig, I. Impact of electron-impurity scattering on the spin relaxation time in graphene: A first-principles study. Phys. Rev. Lett. 2013, 110, 156602. [CrossRef] [PubMed]

30. Soriano, D.; van Tuan, D.; Dubois, S.M.-M.; Gmitra, M.; Cummings, A.W.; Kochan, D.; Ortmann, F.; Charlier, J.-C.; Fabian, J.; Roche, S. Spin transport in hydrogenated graphene. 2D Mater. 2015, 2, 022002. [CrossRef]

31. Tuan, D.V.; Ortmann, F.; Soriano, D.; Valenzuela, S.O.; Roche, S. Pseudospin-driven spin relaxation mechanism in graphene. Nat. Phys. 2014, 10, 857. [CrossRef] 
32. Tuan, D.V.; Ortmann, F.; Cummings, A.W.; Soriano, D.; Roche, S. Spin dynamics and relaxation in graphene dictated by electron-hole puddles. Sci. Rep. 2016, 6, 21046. [CrossRef] [PubMed]

33. Cummings, A.W.; Roche, S. Effects of dephasing on spin lifetime in ballistic spin-orbit materials. Phys. Rev. Lett. 2016, 116, 086602. [CrossRef] [PubMed]

34. Wojtaszek, M.; Vera-Marun, I.J.; Maassen, T.; van Wees, B.J. Enhancement of spin relaxation time in hydrogenated graphene spin-valve devices. Phys. Rev. B 2013, 87, 081402. [CrossRef]

35. Guimarães, M.H.; Veligura, A.; Zomer, P.J.; Maassen, T.; Vera-Marun, I.J.; Tombros, N.; van Wees, B.J. Spin Transport in High-Quality Suspended Graphene Devices. Nano Lett. 2012, 12, 3512-3517. [CrossRef] [PubMed]

36. Ingla-Aynés, J.; Guimarães, M.H.D.; Meijerink, R.J.; Zomer, P.J.; van Wees, B.J. 24- $\mu$ m spin relaxation length in boron nitride encapsulated bilayer graphene. Phys. Rev. B 2015, 92, 201410(R) . [CrossRef]

37. Bychkov, Y.A.; Rashba, E.I. Properties of a 2D electron gas with lifted spectral degeneracy. JEPT Lett. 1984, $39,78$.

38. Wu, M.W.; Jiang, J.H.; Weng, M.Q. Spin dynamics in semiconductors. Phys. Rep. 2010, 493, 61-236. [CrossRef]

39. Huang, C.; Chong, Y.D.; Cazalilla, M.A. Direct coupling between charge and spin polarization by extrinsic mechanisms in graphene. Phys. Rev. B 2016, 94, 085414. [CrossRef]

40. Huang, C.; Chong, Y.D.; Cazalilla, M.A. Anomalous Nonlocal Resistance and Spin-Charge Conversion Mechanisms in Two-Dimensional Metals. Phys. Rev. Lett. 2017, 119, 136804. [CrossRef] [PubMed]

41. Ochoa, H.; Neto, A.H.C.; Guinea, F. Elliot-Yafet mechanism in graphene. Phys. Rev. Lett. 2012, 108, 206808. [CrossRef] [PubMed]

42. Zhang, P.; Wu, M.W. Electron spin relaxation in graphene with random Rashba field: Comparison of the D'yakonov-Perel'and Elliott-Yafet-like mechanisms. New J. Phys. 2012, 14, 033015. [CrossRef]

43. Milletarì, M.; Offidani, M.; Ferreira, A.; Raimondi, R. Covariant conservation laws and the spin Hall effect in Dirac-Rashba systems. Phys. Rev. Lett. 2017, 119, 246801. [CrossRef] [PubMed]

44. Offidani, M.; Milletarì, M.; Raimondi, R.; Ferreira, A. Optimal Charge-to-Spin Conversion in Graphene on Transition-Metal Dichalcogenides. Phys. Rev. Lett. 2017, 119, 196801. [CrossRef] [PubMed]

45. Dimitrova, O.V. Spin-Hall conductivity in a two-dimensional Rashba electron gas. Phys. Rev. B 2005, 71, 245327. [CrossRef]

46. Raimondi, R.; Gorini, C.; Schwab, P.; Dzierzawa, M. Quasiclassical approach to the spin Hall effect in the two-dimensional electron gas. Phys. Rev. B 2006, 74, 035340. [CrossRef]

47. Raimondi, R.; Schwab, P.; Gorini, C.; Vignale, G. Spin-orbit interaction in a two-dimensional electron gas: A SU(2) formulation. Ann. Phys. 2012, 524, 153-162. [CrossRef]

48. Maleki, A.; Raimondi, R. Inverse Spin Galvanic Effect in the Presence of Impurity Spin-Orbit Scattering: A Diagrammatic Approach. Condens. Matter 2017, 2, 17.

49. Gorini, C.; Sheikhabadi, A.M.; Shen, K.; Tokatly, I.V.; Vignale, G.; Raimondi, R. Theory of current-induced spin polarization in an electron gas. Phys. Rev. B 2017, 95, 205424. [CrossRef]

50. The ordering of the basis is $(\mathbf{A}, \mathbf{B})^{\mathrm{t}}$, where $\mathbf{A}=\left(A^{\uparrow}, A^{\downarrow}\right)$ includes up and down spin states on sublattice site $A$ (similarly for $B$ ). We use natural units where $\hbar \equiv 1 \equiv e$, unless stated otherwise.

51. Schwab, P.; Raimondi, R.; Gorini, C. Spin-charge locking and tunneling into a helical metal. EPL 2011, 93, 67004. [CrossRef]

52. Hsieh, D.; Xia, Y.; Qian, D.; Wray, L.; Dil, J.H.; Meier, F.; Osterwalder, J.; Patthey, L.; Checkelsky, J.G.; Ong, N.P.; et al. A tunable topological insulator in the spin helical Dirac transport regime. Nature 2009, 460, 1101. [CrossRef] [PubMed]

53. Brosco, V.; Benfatto, L.; Cappelluti, E.; Grimaldi, C. Unconventional dc transport in Rashba electron gases. Phys. Rev. Lett. 2016, 116, 166602. [CrossRef] [PubMed]

54. Milletarì, M.; Ferreira, A. Quantum diagrammatic theory of the extrinsic spin Hall effect in graphene. Phys. Rev. B 2016, 94, 134202. [CrossRef]

55. Ferreira, A.; Viana-Gomes, J.; Nilsson, J.; Mucciolo, E.R.; Peres, N.M.R.; Neto, A.H.C. Unified description of the dc conductivity of monolayer and bilayer graphene at finite densities based on resonant scatterers. Phys. Rev. B 2011, 83, 165402. [CrossRef]

56. Ferreira, A.; Rappoport, T.G.; Cazalilla, M.A.; Neto, A.H.C. Extrinsic spin Hall effect induced by resonant skew scattering in graphene. Phys. Rev. Lett. 2014, 112, 066601. [CrossRef] [PubMed] 
57. Chen, J.H.; Cullen, W.G.; Jang, C.; Fuhrer, M.S.; Williams, E.D. Defect scattering in graphene. Phys. Rev. Lett. 2009, 102, 236805. [CrossRef] [PubMed]

58. Monteverde, M.; Ojeda-Aristizabal, C.; Weil, R.; Bennaceur, K.; Ferrier, M.; Guéron, S.; Glattli, C.; Bouchiat, H.; Fuchs, J.N.; Maslov, D.L. Transport and elastic scattering times as probes of the nature of impurity scattering in single-layer and bilayer graphene. Phys. Rev. Lett. 2010, 104, 126801. [CrossRef] [PubMed]

59. Ni, Z.H.; Ponomarenko, L.A.; Nair, R.R.; Yang, R.; Anissimova, S.; Grigorieva, I.V.; Schedin, F.; Blake, P.; Shen, Z.X.; Hill, E.H.; et al. On resonant scatterers as a factor limiting carrier mobility in graphene. Nano Lett. 2010, 10, 3868-3872. [CrossRef] [PubMed]

60. Katoch, J.; Chen, J.-H.; Tsuchikawa, R.; Smith, C.W.; Mucciolo, E.R.; Ishigami, M. Uncovering the dominant scatterer in graphene sheets on $\mathrm{SiO}_{2}$. Phys. Rev. B 2010, 82, 081417(R) . [CrossRef]

61. Burkov, A.A.; Nunez, A.S.; MacDonald, A.H. Theory of spin-charge-coupled transport in a two-dimensional electron gas with Rashba spin-orbit interactions. PRB 2004, 70, 155308. [CrossRef]

62. Burkov, A.A.; Balents, L. Spin relaxation in a two-dimensional electron gas in a perpendicular magnetic field. Phys. Rev. B. 2004, 69, 245312 . [CrossRef]

63. Shen, K.; Vignale, G.; Raimondi, R. Microscopic theory of the inverse Edelstein effect. Phys. Rev. Lett. 2014, 112, 096601. [CrossRef] [PubMed]

64. Rammer, J. Quantum Transport Theory; Taylor \& Francis Inc.: Abingdon, UK, 1998.

65. McCann, E.; Kechedzhi, K.; Fal'ko, V.I.; Suzuura, H.; Ando, T.; Altshuler, B.L. Weak-localization magnetoresistance and valley symmetry in graphene. Phys. Rev. Lett. 2006, 97, 146805. [CrossRef] [PubMed]

66. Ostrovsky, P.M.; Gornyi, I.V.; Mirlin, A.D. Electron transport in disordered graphene. Phys. Rev. B 2006, 74, 235443. [CrossRef]

67. Attention must be paid on this point when considering space-dependent dynamics. In fact, while spininjection can be performed locally, electric field lines extending in space can generate spin nonlocally, i.e., far away from the source $[40,68]$. However this circumstance is irrelevant for our purpose of obtaining the SRTs.

68. Abanin, D.A.; Shytov, A.V.; Levitov, L.S.; Halperin, B.I. Nonlocal charge transport mediated by spin diffusion in the spin Hall effect regime. Phys. Rev. B 2009, 79, 03504. [CrossRef]

69. Ado, I.A.; Dmitriev, I.A.; Ostrovsky, P.M.; Titov, M. Anomalous Hall effect with massive Dirac fermions. EPL 2015, 111, 37004. [CrossRef]

70. Milletarì, M.; Ferreira, A. Crossover to the anomalous quantum regime in the extrinsic spin Hall effect of graphene. Phys. Rev. B 2016, 94, 201402(R) . [CrossRef]

71. McCann, E.; Falko, V. $z \rightarrow-z$ Symmetry of Spin-Orbit Coupling and Weak Localization in Graphene. Phys. Rev. Lett. 2012, 108, 166606. [CrossRef] [PubMed]

72. Cummings, A.W.; Garcia, J.H.; Fabian, J.; Roche, S. Giant Spin Lifetime Anisotropy in Graphene Induced by Proximity Effects. Phys. Rev. Lett. 2017, 119, 206601. [CrossRef] [PubMed]

73. Ghiasi, T.S.; Ingla-Aynes, J.; Kaverzin, A.A.; van Wees, B.J. Large Proximity-Induced Spin Lifetime Anisotropy in Transition-Metal Dichalcogenide/Graphene Heterostructures. Nano Lett. 2017, 17, 7528-7532. [CrossRef] [PubMed]

74. Benítez, L.A.; Sierra, J.F.; Torres, W.S.; Arrighi, A.; Bonell, F.; Costache, M.V.; Valenzuela, S.O. Strongly anisotropic spin relaxation in graphene-transition metal dichalcogenide heterostructures at room temperature. Nat. Phys. 2017, 14, 303. [CrossRef]

75. Wakamura, T.; Reale, F.; Palczynski, P.; Guéron, S.; Mattevi, C.; Bouchiat, H. Strong Anisotropic Spin-Orbit Interaction Induced in Graphene by Monolayer $\mathrm{WS}_{2}$. Phys. Rev. Lett. 2018, 120, 106802. [CrossRef] [PubMed]

Sample Availability: This paper complies with EPSRC requirements on data management. The theoretical research described here is not based upon data generated by the authors in the course of EPSRC-funded research.

(C) 2018 by the authors. Licensee MDPI, Basel, Switzerland. This article is an open access article distributed under the terms and conditions of the Creative Commons Attribution (CC BY) license (http://creativecommons.org/licenses/by/4.0/). 